\title{
When it's fair to share
}

Humans have at least two characteristics to be proud of. Compared with other great apes, we are not only better collaborators but also more generous. On page 328 of this issue, Hamann et al. explore the connection between these two traits (K. Hamann et al. Nature 476, 328-331; 2011).

The age at which humans first show tendencies towards sharing has been investigated extensively. Three-to-five-yearolds seem to divide any windfall resources unfairly, keeping something they have obtained without effort mostly to themselves. After that, however, they become more inclined to share windfalls equally.

Hamann and colleagues wondered whether children would behave differently if the resource had been obtained through joint effort. They studied pairs of 2- and 3-yearold children when, in each case, one child had greater access to the resources (to three of four toys) and so could choose whether to share them with their partner. The toys could be obtained in three ways: through the collaborative efforts of both children; freely, with no effort by either; or through independent work.

The authors found that 3-year-olds, but not 2 -year-olds, in the position of power opted to share more often (approximately $75 \%$ of the time) after a collaborative effort than after parallel work (about 25\%). So, although the toddlers may not have acquired a sense of 'reward in return for work', they understood that resource distribution should depend on how the resource was obtained.

Is this link between collaboration and fairness uniquely human? If other primates don't appreciate the value of collaboration as much as we do, they would be expected to be indifferent to the idea of allocating resources depending on how they were acquired.

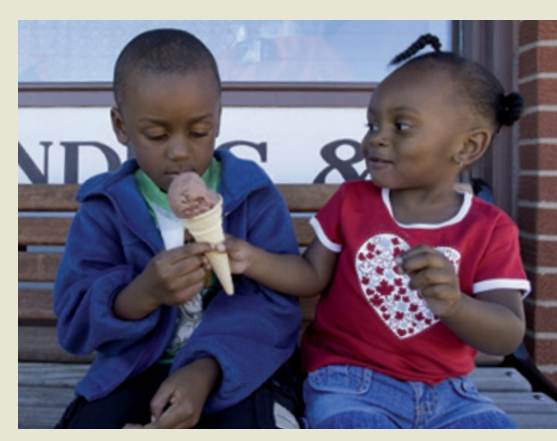

Testing this hypothesis, Hamann et al. found that, when in a position of advantage, chimpanzees sometimes shared, but this was independent of whether a food resource was obtained through collaboration or luck.

An explanation for this behaviour in chimps may be that, unlike in humans, foraging does not depend on collaboration. In other words, humans appreciate the importance of collaboration for survival and so invest in being included in future collaborative efforts by sharing existing resources. Luckily, we seem to learn this quite early on. Sadaf Shadan
- and these enormous clouds have become known simply as Lyman-a blobs.

Although Steidel and colleagues' initial measurements ${ }^{2}$ detected only two bright emission clouds, follow-up work has shown that these are just the tip of the iceberg, with smaller clouds being seen around fainter galaxies. Of course, the key question is, what powers the blobs? If this could be answered for the largest systems, it could provide crucial insight into the formation of galaxies.

Ten years on, the puzzle of the blobs' identity remains. There are two front runners: that they are cooling radiation, released when filamentary gas falls rapidly onto early galaxies; or that the emission is light from a central burst of star formation or a black hole (an active galactic nucleus, AGN) that has been scattered by an extended halo of gas surrounding the system.

The first explanation has been gaining popularity for a while. Theoretical models suggest that galaxies form at the intersection of thin filaments of the 'cosmic web' of dark matter and gas that exist in the Universe. Gas flowing into the galaxies along these filaments must lose energy, providing an ample, distributed source of power ${ }^{3,4}$. Could the Lyman- $\alpha$ blobs be the observational confirmation of this prediction? By contrast, evidence for the second hypothesis is circumstantial. It comes from the frequently observed association ${ }^{5,6}$ of bright Lyman- $\alpha$ blobs with AGNs and starburst galaxies, which form stars at exceptionally high rates. However, Hayes and colleagues' discovery ${ }^{1}$ of polarized emission from the largest and brightest of the blobs now argues strongly in favour of the second option.

To appreciate the significance of this result, it is essential to understand what makes the emission from the blobs special. The emission is seen only at the wavelength corresponding to the Lyman-a electronic transition of hydrogen. The Lyman-a spectral line is created by a transition from the first excited state of hydrogen to the ground state. Because atoms in the ground state are common, the transition is resonant, meaning that a Lyman-a photon that is emitted by one atom is quickly absorbed by a neighbouring atom, promoting it to the excited state. This atom quickly decays to the ground state, releasing another Lyman- $\alpha$ photon, which will be absorbed by another atom, and so on.

It is therefore difficult for a Lyman- $\alpha$ photon to escape unless the medium is very highly ionized or the random motions of the atoms cause the photon to undergo Doppler shifting to a higher or lower frequency, so that the frequency of the photon no longer matches the Lyman- $\alpha$ transition of a hydrogen atom. Although this scattering in frequency leads to the photon's escape, it also leaves an imprint on the polarization of the photon because escaping photons are, on average, scattered perpendicular to their initial direction of travel ${ }^{7}$. A similar process is responsible for polarizing the light reflected from the surface of water, and is why many sunglasses contain polarized lenses. Reducing this complex problem to the simplest level, rings of polarized emission would be expected to be observed if the photons originate from a central source, whereas unpolarized emission would be expected if the source of the photons is widely distributed.

In Hayes and colleagues' technically impressive study ${ }^{1}$ of the emission from the brightest of the Lyman-a blobs, the polarization pattern consists of concentric rings around the likely centre of the emission (Fig. 1), in good agreement with the predictions for scattering from a central source. If this result can be generalized to all Lyman-a blobs, it opens up the intriguing possibility of using the properties of the scattered radiation to probe the gas haloes of young galaxies. When combined with absorption measurements from background galaxies and AGNs, observations of extended Lyman- $\alpha$ blobs suggest a promising route to studying the previously invisible extended circum-galactic medium of galaxies ${ }^{8}$. This possibility is exciting, because stars and cold gas account for only a small fraction of the ordinary matter in the Universe: theoretical models predict that most ordinary matter will reside in an extended and hitherto undetectable reservoir.

Richard Bower is at the Institute for Computational Cosmology, Department of Physics, University of Durham, Durham DH1 3LE, UK.

e-mail:r.g.bower@durham.ac.uk

1. Hayes, M., Scarlata, C. \& Siana, B. Nature 476, 304-307 (2011).

2. Steidel, C. C. et al. Astrophys. J. 532, 170-182 (2000).

3. Fardal, M. A. et al. Astrophys. J. 562, 605-617 (2001).

4. Goerdt, T. et al. Mon. Not. R. Astron. Soc. 407, 613-631 (2010).

5. Matsuda, Y. et al. Astron. J. 128, 569-584 (2004).

6. Geach, J. E. et al. Astrophys. J. 700, 1-9 (2009).

7. Dijkstra, M. \& Loeb, A. Mon. Not. R. Astron. Soc. 386 , 492-504 (2008).

8. Steidel, C. C. et al. Astrophys. J. 736, 160 (2011). 